\title{
Application of Gold Nanoparticles of Different Concentrations to Improve the Therapeutic Potential of Autologous Conditioned Serum: Potential Implications for Equine Regenerative Medicine
}

\author{
Krzysztof Marycz, ${ }^{1}$ Jakub Kolankowski, ${ }^{1}$ Jakub Grzesiak, ${ }^{1,2}$ \\ Mateusz Hecold, ${ }^{3}$ Olga Rac, ${ }^{4}$ and Helena Teterycz ${ }^{4}$ \\ ${ }^{1}$ Electron Microscopy Laboratory, Wroclaw University of Environmental and Life Sciences, 51-631 Wroclaw, Poland \\ ${ }^{2}$ Department of Biotechnology, EIT+ Wroclaw Research Center, 54-066 Wroclaw, Poland \\ ${ }^{3}$ Department of Large Animal Diseases with Clinic, Faculty of Veterinary Medicine, Warsaw University of Life Sciences, \\ 02-776 Warsaw, Poland \\ ${ }^{4}$ Faculty of Microsystem Electronics and Photonics, Wroclaw University of Technology, 50-372 Wroclaw, Poland
}

Correspondence should be addressed to Krzysztof Marycz; krzysztofmarycz@interia.pl

Received 18 May 2015; Revised 29 June 2015; Accepted 30 June 2015

Academic Editor: Subrata Kundu

Copyright (C) 2015 Krzysztof Marycz et al. This is an open access article distributed under the Creative Commons Attribution License, which permits unrestricted use, distribution, and reproduction in any medium, provided the original work is properly cited.

\begin{abstract}
The aim of this work was to evaluate the effect of different concentrations of gold nanoparticles (AuNPS) on equine platelet morphology, ultrastructure as well as secretion of bone morphogenetic protein 2 (BMP-2), vascular endothelial growth factor (VEGF), gelsolin (GSN), and tumor necrosis factor alpha (TNF- $\alpha$ ). The gold nanoparticles, with an average diameter of $20 \mathrm{~nm}$, were incubated with whole anticoagulated blood at $1 \%, 5 \%$, and $10 \% \mathrm{w} / \mathrm{v}$ for 24 hours. We showed that the application of gold nanoparticles at all the tested concentrations led to platelet activation; however, 5\% of AuNPS resulted in the secretion of elevated levels of GSN and VEGF, while the concentration of TNF- $\alpha$ was the lowest. The data indicate that this method may be considered a promising tool for improving the currently used in clinical practice commercially available autologous conditioned serum (ACS) isolation systems for the treatment of equine osteoarthritis.
\end{abstract}

\section{Introduction}

In the last decade, many different biotechnological solutions on the basis of autologous factors have been proposed for the treatment of musculoskeletal system disorders of horses. These include application of autologous or/and allogenic stem cell therapy, conditioned autologous serum (IRAP/Orthokin), growth factors, and platelet-rich plasma (PRP) $[1,2]$. Growing evidence indicates a positive clinical effect of PRP in the treatment of various equine musculoskeletal disorders. However, little is known about the possible platelet (PLT) enhancement with the use of external molecules in the context of platelet degranulation. Among the most important functions of PLT are the initiation of coagulation, fibrinolysis, and vasoconstriction. The PLT function is closely correlated with their morphology and ultrastructure. PLT are the smallest cellular blood components with a diameter of $2-3 \mathrm{~mm}$, which are present in the circulatory system in an inactive form. When activated, PLT change their morphotype from discoidal to flat and cuboidal shape. Regarding the ultrastructure, PLT are characterized by the presence of electron dense cell membrane, $\alpha$-granules, $\delta$ granules, mitochondria, lysosomes, and peroxisomes. All of them are involved in various ways in the process known in the literature as the "PLT activation process." PLT are primarily activated during the process of wound healing by contact with collagen exposed to the bloodstream after endothelial injury and secreted intercellular mediators that stimulate 
the regenerative process. PLT secrete stored intercellular mediators and cytokines from the cytoplasmic pool and release their $\alpha$-granule content after aggregation. Among the most important known factors are transforming growth factor (TGF), platelet-derived growth factor (PDGF), insulinlike growth factor (IGF), fibroblast growth factor (FGF), vascular endothelial growth factor (VEGF), and bone morphogenic protein 2 (BMP-2). These proteins exert a paracrine effect on different cell types, for example, tendon cells [37], myocytes [3], mesenchymal stem cells [8-11], osteoblasts $[3,12,13]$, and fibroblasts [13-16]. Cell proliferation, viability, and differentiation potential are increased when stimulated with the above-listed proteins. However, PLT can release not only positive mediators but also proinflammatory factors, such as serotonin, interleukin-6, or tumor necrosis factor alpha (TNF- $\alpha$ ), which, in addition to immunoenzymatic methods, can be clearly visualized with transmission electron microscopy (TEM) [17]. Hence, seeking efficient methods that selectively activate the secretion of positive mediators by PLT in the course of regenerative process seems to be fully justified.

Increasingly frequently nanomaterial-based technology becomes a part of regenerative medicine in both human and veterinary medicine. A positive effect of biomaterials or scaffold-based biomaterials was shown in the healing of bones or cartilage defects. The synthesis of gold nanoparticles and, more importantly, the possibility of controlling their shape and size by appropriate modifications of synthesis parameters are frequently described topics in the literature due to the broad applicability of these molecules. Gold nanoparticles may be obtained using various methods, such as chemical [18], electrochemical [19], or biological techniques $[20,21]$. The most popular among these methods are the chemical reactions based on the reduction of gold in aqueous solutions. Chloroauric acid is used in these reactions as the precursor of nanoparticles. This method of synthesis allows us to modify the shape and size of nanoparticles by adjusting the process parameters [22].

The first article concerning the synthesis of gold nanoparticles was published in 1857. Faraday described in this work the synthesis of gold nanoparticles and observed their red color [23]. Currently, this technique is still commonly applied. Similar synthesis can be carried out both in aqueous and nonaqueous solvents, and the reducing agents applied include citric acid, ethanol, formaldehyde, or sodium borohydride [24]. Nanoparticles tend to agglomerate because of their high surface energy. For this reason, nanoparticle stabilizers are added during the colloid preparations, which prevent the agglomeration. With respect to gold nanoparticles, thiols are commonly used stabilizers because of the strong interaction between gold and thiol ions [25-27]. Strong Au-S bonds can also be obtained when using polyethylene glycol (PEG) functionalized with thiol groups [28]. Polyethylenimine (PEI) is another commonly used stabilizer of gold colloids. The size of the synthesized nanoparticles may be regulated by modifying the $\mathrm{pH}$ of solutions containing a stabilizer [29]. Moreover, PEI serves as a reductive agent during the synthesis $[18,30]$. However, the most frequently used polymer for stabilizing the water colloids of gold is polyvinylpyrrolidone
(PVP) [31-33]. It can be used both as a stabilizer and a reductive agent if the synthesis of gold nanoparticles is performed in aqueous solution. In most cases, however, PVP is used only as a stabilizer [34, 35].

Nanoparticles, not only of gold, are increasingly applied in medicine. Therefore, the search for biocompatible stabilizers is necessary. One such substance is poly-L-lysine (PLL), which tends to create micelles in water; thus, it may be used as a polymer-stabilizing agent. Gold nanoparticles stabilized with methoxypolyethylene glycol-graft-poly(L-lysine) copolymer (MPEG-gPLL) may find their biomedical application in noninvasive neoplasm therapy involving electromagnetic radiation [36].

Gold nanoparticles with the attached PLL molecules can be embedded in porous silica grains [37], PLL, and hyaluronic acid-based films [38] or may be bound with PLL chains, which results in covalently bonded gold nanoparticles/polyL-lysine hybrid material [39].

Recently, a positive clinical effect of autologous conditioned serum in the treatment of different lamenessassociated equine diseases using a commercially available gold nanoparticle-based system (GOLDIC) has been demonstrated. Our previous study has shown beneficial effects of this technology on osteogenic and chondrogenic differentiation potential of adipose-derived mesenchymal/stromal stem cells in vitro (ASCs) [40]. Therefore, we were interested whether gold nanoparticles of different size and concentration would affect the secretion of proregenerative factors by PLT with simultaneous inhibition of proinflammatory cytokines. The study demonstrated that AuNPS, at a specific concentration, enhanced the secretion of BMP-2, VEGF, and IL-6, while they in parallel inhibited the secretion of TNF- $\alpha$.

\section{Materials and Methods}

2.1. Production of Gold Nanoparticles. Poly-L-lysine (PLL) with an average molecular weight of $4,700 \mathrm{~g} / \mathrm{mol}$ was purchased from Sigma-Aldrich. Aqueous solutions of the polymer were prepared by weighing the solid material and dissolving it in water at room temperature. Polymer aqueous solutions were prepared at a concentration of $128 \mathrm{~g} / \mathrm{L}$, which corresponded to $1 \mathrm{M}$, as calculated by the molar mass of the monomer per liter. Aqueous solution of chloroauric acid $\mathrm{HAuCl}_{4}(\mathrm{POCH}$, Poland), at a concentration of $0.04 \mathrm{M}$, was used as a precursor of gold nanoparticles. Aqueous solution of ascorbic acid (0.2 M, POCH, Poland) was used as a reducing agent. A mixture of equal volumes of both compounds was prepared directly before the synthesis.

Synthesis of nanoparticles was carried out using a liquid chemical method of chloroauric acid reduction with a reducing mixture in the presence of PLL. Gold nanoparticles were obtained by mixing $250 \mathrm{~mL}$ of water with $625 \mu \mathrm{L}$ of chloroauric acid, $20 \mathrm{~mL}$ of reducing mixture, and $20 \mu \mathrm{L}$ of PLL at room temperature. The concentration of gold nanoparticles in the colloid was $50 \mathrm{ppm}$.

The electrical conductance of water used for synthesis was $0.2 \mu \mathrm{S} / \mathrm{cm}$, and the water containing PLL and chloroauric acid was $316.7 \mu \mathrm{S} / \mathrm{cm}$, while the colloid (after the addition of 
reducing agent) containing the AuNPS was $184.2 \mu \mathrm{S} / \mathrm{cm}$. The $\mathrm{pH}$ of final AuNPS colloid was 3.5.

2.2. Characterization of Gold Nanoparticles. The absorption (A) of nanogold colloid solution was measured with a QE65000 spectrophotometer (Ocean Optics). The absorption spectrum was measured at a wavelength of 200 to $700 \mathrm{~nm}$ in a quartz cuvette.

Dynamic light scattering (DLS) was used to determine the size and distribution profile of Au particles in the dispersion using NICOMP 380 Particle Sizing System (Nicomp, Santa Barbara, USA). This technique can be used to determine the size distribution profile of small particles in colloid solution.

The analysis of metallic nanoparticle microstructures was performed with a transmission electron microscope (TEM, FEI Tecnai G2 20 X-TWIN).

2.3. Incubation of Equine Blood with Gold Nanoparticles. Whole peripheral blood from 2 healthy horses was collected into the blood container with CPDA anticoagulant. Blood was transferred to two sets of centrifuge tubes $(n=2)$ containing AuNPS solution at different volumes, giving in final $1 \%, 5 \%$, and $10 \% \mathrm{v} / \mathrm{v}$ (gold solution/whole blood) of gold nanoparticles. The second set served as a control group. The tubes with the blood from both groups were incubated at $37^{\circ} \mathrm{C}$ for $24 \mathrm{~h}$ to activate platelets. After this time, the blood was centrifuged at $300 \times \mathrm{g}$ for 10 minutes to separate blood cells from plasma. Subsequently, the plasma was collected to new tubes and centrifuged at $640 \times \mathrm{g}$ to separate plateletrich plasma from platelet-poor plasma. The supernatants were collected to determine BMP-2, VEGF, IL-6, and TNF- $\alpha$ concentrations, while the platelets were suspended and fixed in $2.5 \%$ glutaraldehyde for one hour at room temperature.

2.4. Morphological and Ultrastructural Analysis of Platelets. Fixed platelets were centrifuged at $640 \times \mathrm{g}$ for $10 \mathrm{~min}$. The supernatant (glutaraldehyde) was removed and the pellet was washed three times using Sorensen's buffer and once with ultrapure distilled water. The platelets were centrifuged after each washing. Subsequently, platelets were dehydrated in a graded series of ethyl alcohol. Then, the material suspended in $99.8 \%$ of ethanol was smeared on a glass slide and allowed to dry. The samples were coated with gold and observed using a scanning electron microscope at $10 \mathrm{kV}$ (EVO LS 15, Zeiss).

2.5. Scanning Transmission Electron Microscopy. Each subsequent step of preparation was preceded by spinning down the samples. The prepared platelets were fixed with $2.5 \%$ glutaraldehyde for 30 minutes. After the fixation, the platelets were washed three times with filtered distilled water. The washed platelets were postfixed with $2 \%$ osmium tetroxide solution for 90 minutes, washed again three times with filtered distilled water, and dehydrated in a graded acetone series. The samples were infiltrated with a 1:1 mixture of Agar low viscosity resin and acetone followed by $100 \%$ Agar. The samples were embedded in Agar low viscosity resin and allowed to polymerize at $60^{\circ} \mathrm{C}$. Ultrathin sections were obtained using an ultramicrotome with a diamond knife,

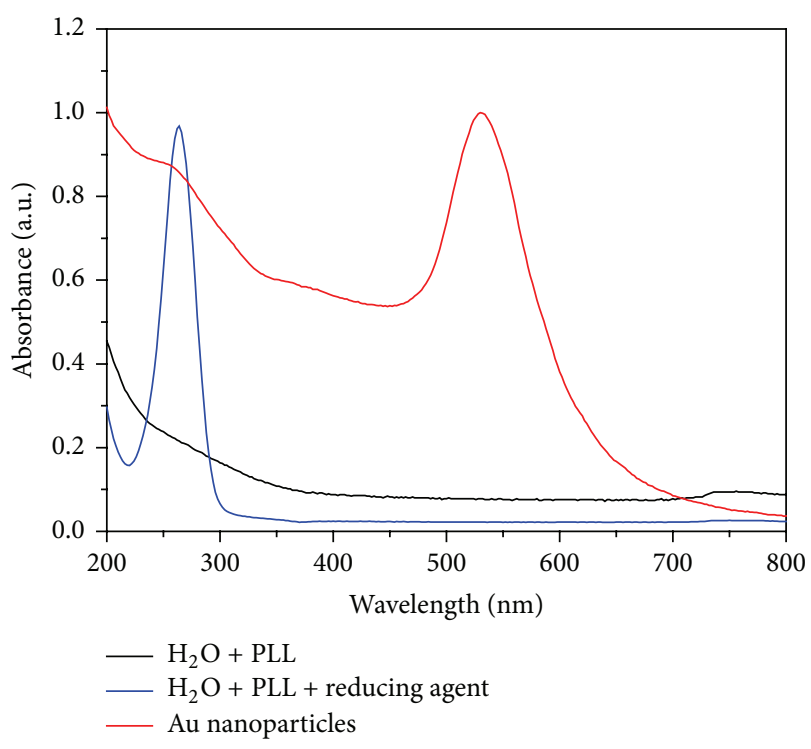

FIgURE 1: UV-Vis spectrum of the gold colloid.

stained with lead citrate and uranyl acetate and observed using a FE-SEM, Auriga60, Zeiss.

2.6. Detection of BMP-2, VEGF, TNF- $\alpha$, and IL-6 in Equine $P R P$. ELISA assays were performed in order to quantitatively analyze the levels of BMP-2, VEGF, TNF- $\alpha$, and p53 in the PRP incubated with different concentrations of gold nanoparticles. All ELISA kits were purchased from MyBiosource Inc., San Diego, CA, except for TNF- $\alpha$, which was purchased from $\mathrm{R} \& \mathrm{D}$ systems. The procedures were performed according to the instructions provided by the vendor.

\section{Results}

3.1. Characterization of Gold Nanoparticles. Spectrophotometric measurements in the range of $200-800 \mathrm{~nm}$ confirmed the presence of gold nanoparticles in the solution. The peak observed at $530 \mathrm{~nm}$ wavelength was characteristic of gold nanoparticles (Figure 1). An additional peak at a $250 \mathrm{~nm}$ wavelength was derived from additional substrates, like PLL. The average size of nanoparticles, determined using DLS, was $8 \mathrm{~nm}$ (Figure 2).

TEM analysis of gold nanoparticles showed that the size of the obtained nanoparticles was approximately $20 \mathrm{~nm}$. The nanoparticles were of polygonal shape, and the majority had hexagonal structure (Figure 3). EDX analysis confirmed that precursor molecules were the only particles in the investigated solution (Figure 4).

3.2. Morphology and Ultrastructure of Platelets. The platelets of all groups showed significant differences in their morphology and activation stage. Platelets incubated with a $1 \%$ solution of AuNPS were at a later stage of activation, with visible single pseudopodia, but without signs of aggregation (Figures 5(a) and 5(b)). Ultrastructural analysis of PLT from 


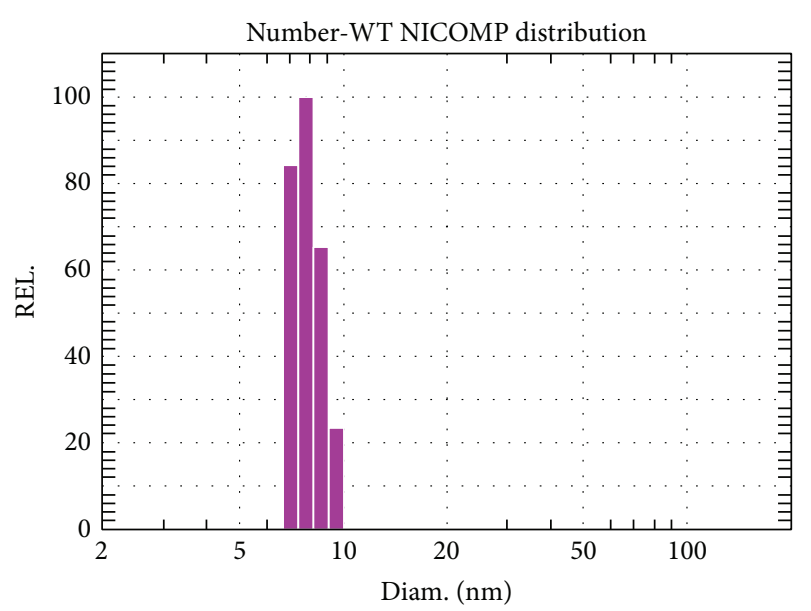

FIGURE 2: DLS results of nanoparticle diameter distribution.

$1 \%$ group confirmed the weak activation of platelets by visible pseudopodia and partially released granules (Figures 6(a) and 6(b)). Platelets incubated with 5\% AuNPS solution showed a higher level of activation, as numerous pseudopodia and moderate aggregation were observed (Figures 5(c) and 5(d)). Transmission electron microscopy revealed a release of a significant quantity of granules and the advanced process of platelet aggregation (Figures 6(c) and 6(d)). The samples incubated with $10 \%$ AuNPS solution were at the latest activation stage; the platelets were mostly aggregated and developed numerous pseudopodia (Figures 5(e) and 5(f)). Scanning electron microscopy confirmed the analysis performed by TEM: the platelets were aggregated and most of the granules were released (Figures 6(e) and 6(f)). Platelets from the control group, which were not incubated with AuNPS, showed low activation, discoid shape, and lack of pseudopodia development (Figures 5(g) and 5(h)). Ultrastructure analysis demonstrated that the majority of granules were still present in the platelets (Figures 6(g) and 6(h)).

3.3. Detection of BMP-2, VEGF, TNF- $\alpha$, and IL-6 in Equine $P R P$. The concentrations of the analyzed factors were significantly different between groups. The concentration of BMP-2 in plasma incubated with $1 \%$ and $5 \%$ of gold nanoparticles was comparable and significantly elevated in relation to control. The level of BMP-2 in the samples incubated with $10 \%$ of AuNPS was also strongly increased and was higher than that in the control and other groups. The concentration of interleukin- 6 was also significantly increased in the experimental groups, with the highest concentration recorded in the samples incubated with 5\% of AuNPS. The presence of TNF- $\alpha$ proinflammatory cytokine was detected only in the samples incubated with $10 \%$ of AuNPS. The level of VEGF was slightly higher in the samples incubated with $1 \%$ of AuNPS, but this difference was not statistically significant. In contrast, the samples incubated in $5 \%$ and $10 \%$ of AuNPS showed a statistically significant increase in the level of this factor (Figure 7).

\section{Discussion}

Autologous conditioned serum (ACS) has been proved to be an effective tool in veterinary medicine in the treatment of musculoskeletal system disorders, including osteoarthritis $(\mathrm{OA})$, which is a slowly progressing degenerative joint disease [41]. Osteoarthritis is characterized by the destruction of articular cartilage, remodeling of the subchondral bone, joint marginal osteophyte formation, and synovitis. The commercially available kits, which are used in equine orthopedics to treat $\mathrm{OA}$, are based on blood cell (mostly platelets) interactions with various types of particles and/or chemicals. It was found that platelets have the unique ability to secrete various anti-inflammatory and proregenerative factors after contact with foreign bodies, including nanoparticles [42]. During the process of degranulation, platelets change their morphology and secrete these factors. However, simultaneously with the secretion of proregenerative proteins, platelets may also release proinflammatory factors, for example, TNF$\alpha$, which is undesirable in the regeneration process. In turn, selective secretion of anti-inflammatory and proregenerative molecules, for example, IL-6, VEGF, or BMP-2 that locally suppress the inflammatory processes and improve the regeneration of damaged tissues, are strongly beneficial in the course of tissue rebuilding. The aforementioned biotechnological strategies, with the exception of stem cell therapy, seem to be increasingly frequently applied by veterinarians. However, still the routine methods of OA treatment are based on the administration of steroidal and nonsteroidal drugs, although these approaches bring only temporary pain relief without the involvement of autologous regenerative processes [43]. The favorable effects of autologous conditioned serum (ACS) application in the clinic have been confirmed in several clinical studies, which further justifies the development of those strategies $[1,44,45]$.

The aim of the present study was to investigate whether the use of different concentrations of gold nanoparticles, incubated with equine blood plasma, will allow us to obtain elevated levels of specific growth factors and proteins in plasma, for example, bone morphogenetic protein 2 (BMP2), vascular endothelial growth factor (VEGF), with simultaneous inhibition of the release of tumor necrosis factor alpha $(\mathrm{TNF}-\alpha)$ from platelets. These factors are well characterized in the literature and their role in regenerative processes in musculoskeletal system disorders is well documented [46].

In the current research, we observed that plasma incubation with various AuNPS concentrations had a significant influence on platelet morphology, ultrastructure, and more importantly, the secretion of BMP-2, VEGF, and IL-6, that is, essential factors in the treatment of musculoskeletal system disorders. On the basis of our observations, when considering the use of AuNPS as an adjunct in the OA treatment, it is important to use structurally and chemically stabile AuNPS.

Aqueous solutions of nanoparticles must include stabilizing agents that protect them from agglomeration. By making the nanoparticles in our laboratory, it was possible to select stabilizing agents for the highest biocompatibility and enhancement of regenerative processes in tissues. Stabilizers used in commercial products are protected by trade 

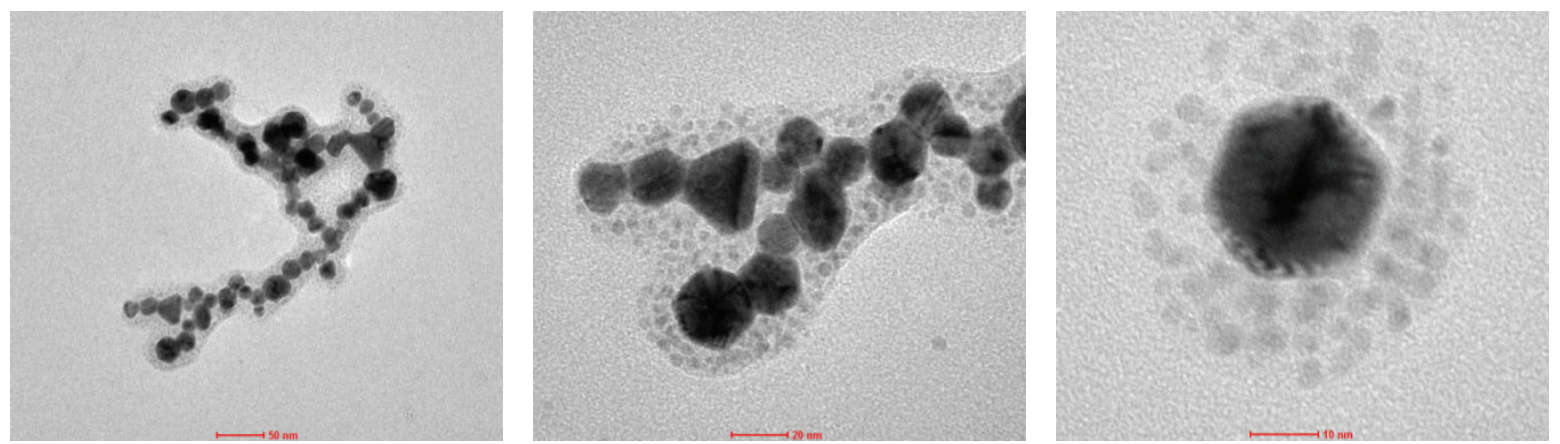

FIGURE 3: TEM images of gold nanoparticles with a stabilizing polymer coating; scale bars are indicated in the images.

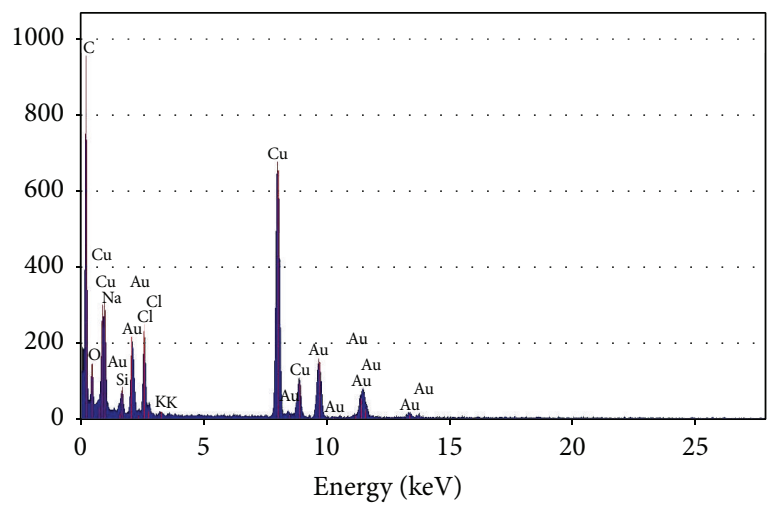

- Acquire EDX

FIGURE 4: EDX analysis of prepared gold nanoparticles.

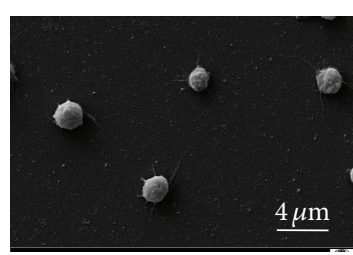

(a)

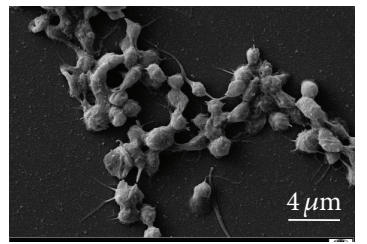

(e)

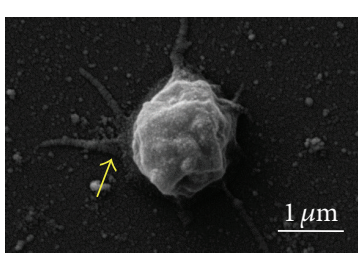

(b)

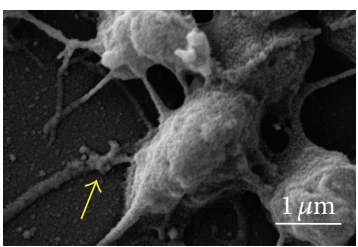

(f)

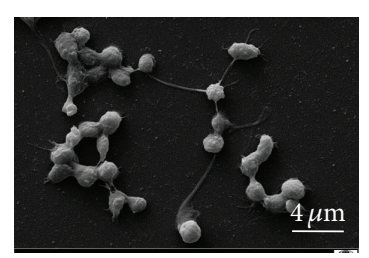

(c)

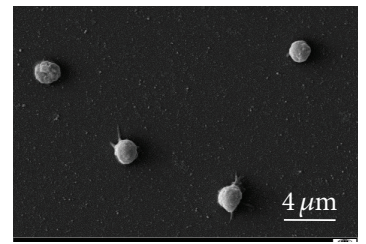

(g)

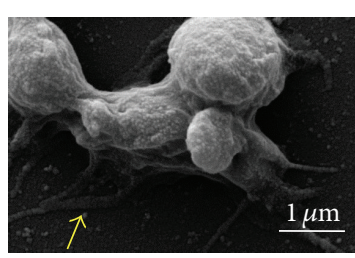

(d)

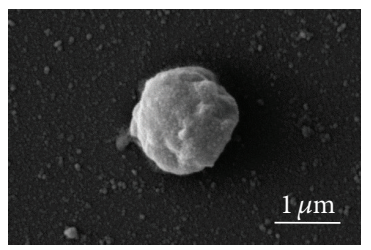

(h)

FIGURE 5: Micrographs depicting the morphology of the platelets incubated with different concentrations of AuNPS and nonincubated control. (a) and (b): 1\% AuNPs; (c) and (d): 5\% AuNPs; (e) and (f): 10\% AuNPs; (g) and (h): control; lamellipodia are marked with yellow arrows; scale bars are indicated in the micrographs.

secret; thus, it is not possible to assess their impact on the results. Selecting the right stabilizer and its most effective concentration allowed us to obtain stable colloids of high concentrations. An additional advantage of the method of $\mathrm{Au}$ nanoparticle synthesis used in this study is the possibility of obtaining colloids of the desired concentration and different particle sizes. Increased diameter of nanoparticles observed in TEM micrographs in relation to results of DLS measurements is caused by the differences in sample preparation procedure. In DLS, samples are measured directly in the water 


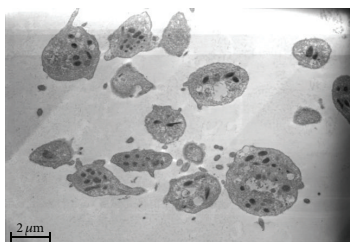

(a)

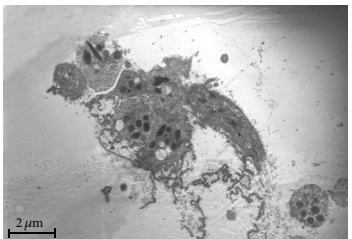

(e)

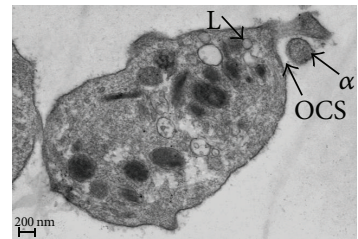

(b)

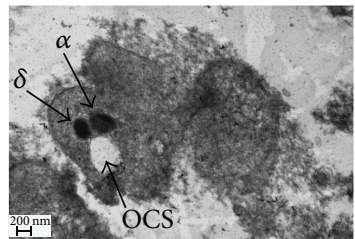

(f)

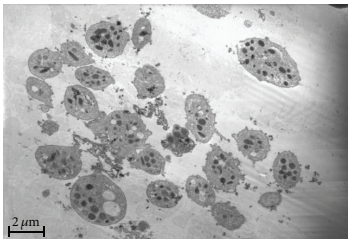

(c)

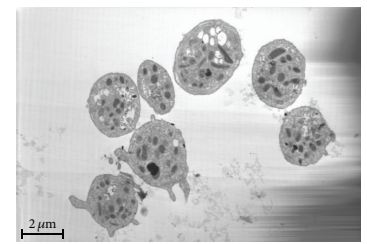

(g)

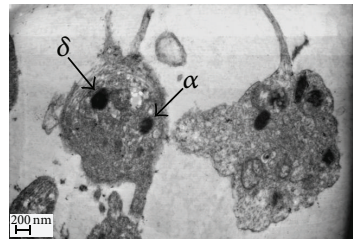

(d)

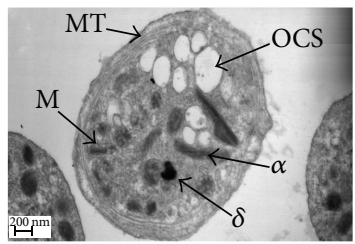

(h)

FIGURE 6: Micrographs presenting the ultrastructure of the platelets incubated with different concentrations of AuNPS and nonincubated control. (a) and (b): 1\% AuNPs; (c) and (d): 5\% AuNPs; (e) and (f): 10\% AuNPs; (g) and (h): control. Organelle abbreviations: $\alpha: \alpha$-granules; $\delta: \delta$-granules; OCS: open canalicular system; M: mitochondria; MT: microtubules; L: lysosomes; scale bars are indicated in the micrographs.
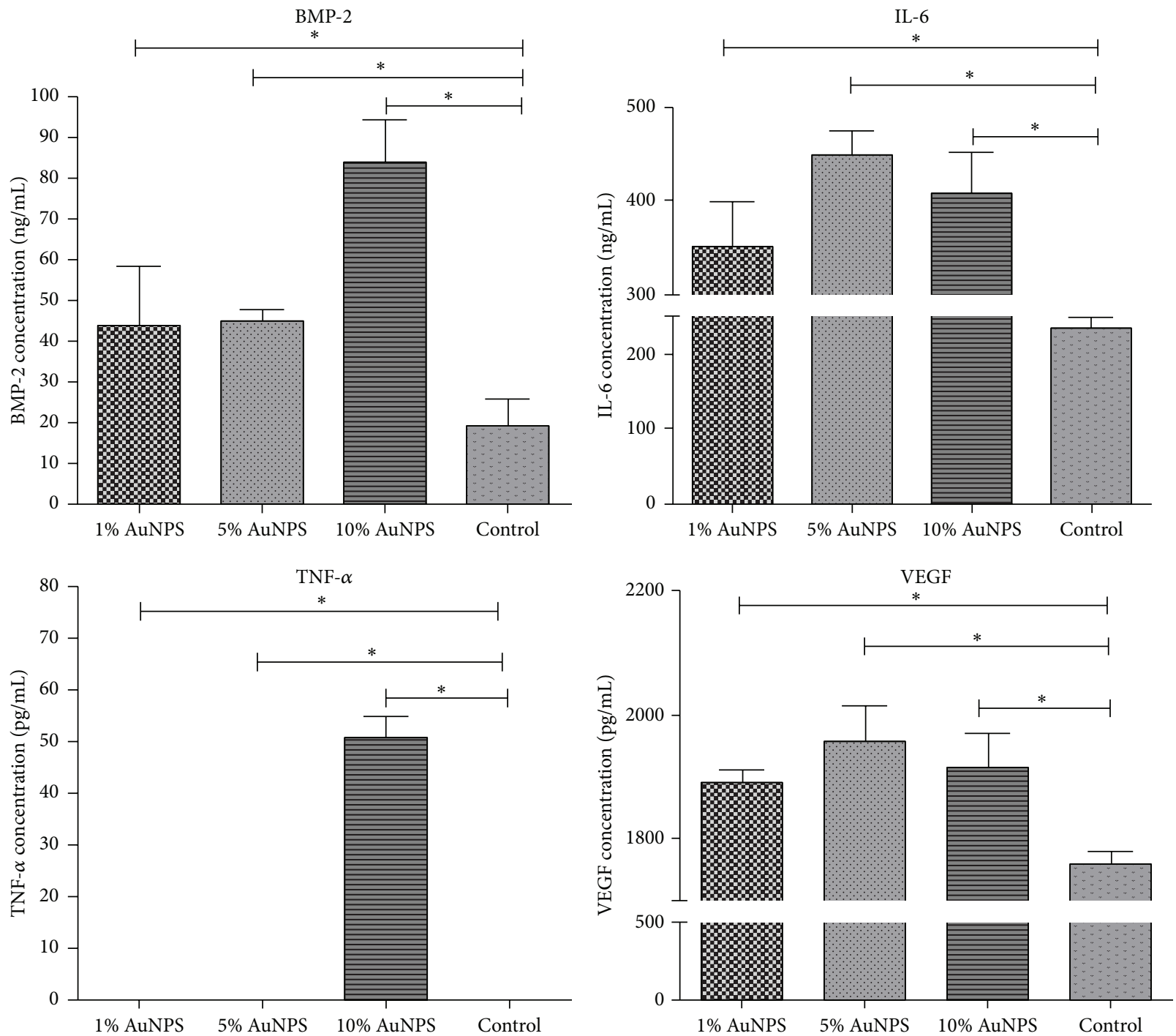

Figure 7: The concentrations of BMP-2, IL-6, TNF- $\alpha$, and VEGF in plasma of the experimental and control groups; ${ }^{*} P<0.05$. 
solution, which protects them from agglomeration, while the colloid sample prepared for TEM had to be dried before observation, which could cause the agglomeration, Ostwald ripening, coalescence, and cluster migration [47]. The process of agglomeration is prominently visible in Figures 3(a) and 3(b), while the coalescence is visible in Figure 3(c).

The current study showed that exposing the blood to 5 and $10 \%$ of AuNPS resulted in the elevated plasma levels of BMP-2, VEGF, and IL-6. The application of 5\% of AuNPS led to secretion of nearly 2-fold grater amount of IL- 6 and BMP-2 than in the control group. In addition, platelets that were exposed to 5\% of AuNPS had a morphology and ultrastructure characteristic for active state, which explained elevated levels of IL-6 and BMP-2 in serum. We have found that platelets exposed to 5\% of AuNPS changed their shape and secreted specific alpha granules, as evidenced by the fibrillar material present in the extracellular spaces. However, numerous cell fusions were also found in the samples with $5 \%$ of AuNPS. Platelets incubated with $1 \%$ of AuNPS developed only single, short processes without signs of aggregation, which is in a good agreement with the levels of analyzed proteins found in plasma. Platelets from control group showed morphology and ultrastructure typical for circulating platelet in an inactive state, with full spectrum of organelles and without signs of pseudopodia development. Thus, the levels of BMP-2, IL-6, and VEGF detected in plasma from control group must have other sources than platelets and should be considered as normal plasma levels. Unfortunately, there is poor data on normal serum levels of these growth factors in healthy horse plasma and their ranges to compare with our results. In studies performed on human material, however, these factors were also detectable in normal serum [48]. Interestingly, the cells exposed to $10 \%$ of AuNPS lost their discoid shape and presented typical cell fusion pattern. In addition, we observed that the cells produced both alpha and dense granules by forming a dense extracellular fibrillar material. What is more, $10 \%$ of AuNPS induced subsequent exocytosis into the extracellular milieu of structures resembling vacuoles containing fibrillar material. This fact also explained the highest concentration of TNF- $\alpha$, which was detected in 10\% AuNPS-treated blood.

To exclude the influence of additional substances present in AuNPS colloid on platelet activation, we performed another test with tested solutions deprived from AuNPS (see Supplementary Material available online at http://dx.doi.org/ 10.1155/2015/521207). Although the PLL or ascorbic acid might induce activation of platelets, their concentration was probably too low in the tested solutions as we did not observe significant number of active platelets in case of this test.

Our findings suggest that the application of different AuNPS concentrations, incubated with whole blood, may be an important factor in obtaining adequate concentrations of various proteins and growth factors. Depending on the AuNPS concentration, platelets secreted various concentrations of proregenerative biomolecules. We showed that $5 \%$ of AuNPS was the optimal concentration to induce the highest levels of IL-6 and VEGF at a markedly lower BMP2 concentration, however, still 2 -fold greater than the control group.

\section{Conclusion}

Our findings indicate that the proper concentration of AuNPS has a significant effect on platelet morphology, ultrastructure, and secretory properties. These findings could potentially be used to optimize the biotechnological systems for the treatment of equine musculoskeletal system disorders.

\section{Conflict of Interests}

The authors declare that there is no conflict of interests regarding the publication of this paper.

\section{Acknowledgment}

The project was supported by Wroclaw Centre of Biotechnology, programme The Leading National Research Centre (KNOW), for years 2014-2018.

\section{References}

[1] P. Wehling, C. Moser, D. Frisbied et al., "Autologous conditioned serum in the treatment of orthopedic diseases," BioDrugs, vol. 21, no. 5, pp. 323-332, 2007.

[2] K. Marycz, N. Y. Toker, J. Grzesiak, K. Wrzeszcz, and P. Golonka, "The therapeutic effect of autogenic adipose derived stem cells combined with autogenic platelet rich plasma in tendons disorders hi horses in vitro and in vivo research," Journal of Animal and Veterinary Advances, vol. 11, no. 23, pp. 4324-4331, 2012.

[3] A. D. Mazzocca, M. B. R. McCarthy, D. M. Chowaniec et al., "The positive effects of different platelet-rich plasma methods on human muscle, bone, and tendon cells," The American Journal of Sports Medicine, vol. 40, no. 8, pp. 1742-1749, 2012.

[4] L. C. Visser, S. P. Arnoczky, O. Caballero, A. Kern, A. Ratcliffe, and K. L. Gardner, "Growth factor-rich plasma increases tendon cell proliferation and matrix synthesis on a synthetic scaffold: an in vitro study," Tissue Engineering A, vol. 16, no. 3, pp. 1021-1029, 2010.

[5] C. H. Jo, J. E. Kim, K. S. Yoon, and S. Shin, "Platelet-rich plasma stimulates cell proliferation and enhances matrix gene expression and synthesis in tenocytes from human rotator cuff tendons with degenerative tears," American Journal of Sports Medicine, vol. 40, no. 5, pp. 1035-1045, 2012.

[6] B. Carofino, D. M. Chowaniec, M. B. McCarthy et al., "Corticosteroids and local anesthetics decrease positive effects of platelet-rich plasma: An in vitro study on human tendon cells," Arthroscopy, vol. 28, no. 5, pp. 711-719, 2012.

[7] M. de Mos, A. E. van der Windt, H. Jahr et al., "Can plateletrich plasma enhance tendon repair? A cell culture study," The American Journal of Sports Medicine, vol. 36, no. 6, pp. 1171-1178, 2008.

[8] H. S. Cho, I. H. Song, S.-Y. Park, M. C. Sung, M.-W. Ahn, and K. E. Song, "Individual variation in growth factor concentrations in platelet-rich plasma and its influence on human mesenchymal stem cells," Korean Journal of Laboratory Medicine, vol. 31, no. 3, pp. 212-218, 2011.

[9] N. Kakudo, T. Minakata, T. Mitsui, S. Kushida, F. Z. Notodihardjo, and K. Kusumoto, "Proliferation-promoting effect of platelet-rich plasma on human adipose-derived stem cells and 
human dermal fibroblasts," Plastic and Reconstructive Surgery, vol. 122, no. 5, pp. 1352-1360, 2008.

[10] A. Mishra, P. Tummala, A. King et al., "Buffered plateletrich plasma enhances mesenchymal stem cell proliferation and chondrogenic differentiation," Tissue engineering. Part C Methods, vol. 15, no. 3, pp. 431-435, 2009.

[11] J. P. Vogel, K. Szalay, F. Geiger, M. Kramer, W. Richter, and P. Kasten, "Platelet-rich plasma improves expansion of human mesenchymal stem cells and retains differentiation capacity and in vivo bone formation in calcium phosphate ceramics," Platelets, vol. 17, no. 7, pp. 462-469, 2006.

[12] O. García-Martínez, C. Reyes-Botella, L. Díaz-Rodríguez et al., "Effect of platelet-rich plasma on growth and antigenic profile of human osteoblasts and its clinical impact," Journal of Oral and Maxillofacial Surgery, vol. 70, no. 7, pp. 1558-1564, 2012.

[13] F. Graziani, S. Ivanovski, S. Cei, F. Ducci, M. Tonetti, and M. Gabriele, "The in vitro effect of different PRP concentrations on osteoblasts and fibroblasts," Clinical Oral Implants Research, vol. 17, no. 2, pp. 212-219, 2006.

[14] E. Anitua, M. Sánchez, M. M. Zalduendo et al., "Fibroblastic response to treatment with different preparations rich in growth factors," Cell Proliferation, vol. 42, no. 2, pp. 162-170, 2009.

[15] S. R. Browning, A. M. Weiser, N. Woolf et al., "Plateletrich plasma increases matrix metalloproteinases in cultures of human synovial fibroblasts," The Journal of Bone and Joint Surgery-American Volume, vol. 94, no. 23, article e172, 2012.

[16] F. Creeper and S. Ivanovski, "Effect of autologous and allogenic platelet-rich plasma on human gingival fibroblast function," Oral Diseases, vol. 18, no. 5, pp. 494-500, 2012.

[17] M. Gawaz, "Role of platelets in coronary thrombosis and reperfusion of ischemic myocardium," Cardiovascular Research, vol. 61, no. 3, pp. 498-511, 2004.

[18] X. Sun, S. Dong, and E. Wang, "One-step preparation of highly concentrated well-stable gold colloids by direct mix of polyelectrolyte and $\mathrm{HAuCl} 4$ aqueous solutions at room temperature," Journal of Colloid and Interface Science, vol. 288, no. 1, pp. 301-303, 2005.

[19] C.-J. Huang, P.-H. Chiu, Y.-H. Wang, K.-L. Chen, J.-J. Linn, and C.-F. Yang, "Electrochemically controlling the size of gold nanoparticles," Journal of the Electrochemical Society, vol. 153, no. 12, pp. D193-D198, 2006.

[20] A. Chauhan, S. Zubair, S. Tufail et al., "Fungus-mediated biological synthesis of gold nanoparticles: potential in detection of liver cancer," International Journal of Nanomedicine, vol. 6, pp. 2305-2319, 2011.

[21] J. Y. Song, H.-K. Jang, and B. S. Kim, "Biological synthesis of gold nanoparticles using Magnolia kobus and Diopyros kaki leaf extracts," Process Biochemistry, vol. 44, no. 10, pp. 1133-1138, 2009.

[22] C.-H. Wang, C.-J. Liu, C.-L. Wang et al., "Optimizing the size and surface properties of polyethylene glycol (PEG)-gold nanoparticles by intense X-ray irradiation," Journal of Physics D: Applied Physics, vol. 41, no. 19, Article ID 195301, 2008.

[23] M. Faraday, "Experimental relations of gold (and other metals) to light," Philosophical Transactions of the Royal Society of London, vol. 147, pp. 145-181, 1857.

[24] M. L. Juan, M. Righini, and R. Quidant, "Plasmon nano-optical tweezers," Nature Photonics, vol. 5, no. 6, pp. 349-356, 2011.

[25] M.-C. Daniel and D. Astruc, "Gold nanoparticles: assembly, supramolecular chemistry, quantum-size-related properties, and applications toward biology, catalysis, and nanotechnology," Chemical Reviews, vol. 104, no. 1, pp. 293-346, 2004.
[26] M. Giersig and P. Mulvaney, "Preparation of ordered colloid monolayers by electrophoretic deposition," Langmuir, vol. 9, no. 12, pp. 3408-3413, 1993.

[27] M. Brust, M. Walker, D. Bethell, D. J. Schiffrin, and R. Whyman, "Synthesis of thiol-derivatised gold nanoparticles in a twophase liquid-liquid system," Journal of the Chemical Society, Chemical Communications, no. 7, pp. 801-802, 1994.

[28] K. Rahme, L. Chen, R. G. Hobbs, M. A. Morris, C. O’Driscoll, and J. D. Holmes, "PEGylated gold nanoparticles: polymer quantification as a function of PEG lengths and nanoparticle dimensions," RSC Advances, vol. 3, no. 17, pp. 6085-6094, 2013.

[29] F. S. Mohammed, S. R. Cole, and C. L. Kitchens, "Synthesis and enhanced colloidal stability of cationic gold nanoparticles using polyethyleneimine and carbon dioxide," ACS Sustainable Chemistry and Engineering, vol. 1, no. 7, pp. 826-832, 2013.

[30] S. Wen, F. Zheng, M. Shen, and X. Shi, "Synthesis of polyethyleneimine-stabilized gold nanoparticles for colorimetric sensing of heparin," Colloids and Surfaces A: Physicochemical and Engineering Aspects, vol. 419, pp. 80-86, 2013.

[31] H. Wang, X. Qiao, J. Chen, X. Wang, and S. Ding, "Mechanisms of PVP in the preparation of silver nanoparticles," Materials Chemistry and Physics, vol. 94, no. 2-3, pp. 449-453, 2005.

[32] S. Boopathi, S. Senthilkumar, and K. L. Phani, "Facile and one pot synthesis of gold nanoparticles using tetraphenylborate and polyvinylpyrrolidone for selective colorimetric detection of mercury ions in aqueous medium," Journal of Analytical Methods in Chemistry, vol. 2012, Article ID 348965, 6 pages, 2012.

[33] J. Nishino and Y. Kanno, "An influence of concentration of polyvinylpyrrolidone on the morphology of silver metal formed from $\mathrm{AgNO}_{3}$ aqueous solution," Journal of Nanomaterials, vol. 2008, Article ID 592838, 5 pages, 2008.

[34] C. E. Hoppe, M. Lazzari, I. Pardiñas-Blanco, and M. A. LópezQuintela, "One-step synthesis of gold and silver hydrosols using poly(N-vinyl-2- pyrrolidone) as a reducing agent," Langmuir, vol. 22, no. 16, pp. 7027-7034, 2006.

[35] M. H. Ullah, T. Hossain, and C.-S. Ha, "Kinetic studies on water-soluble gold nanoparticles coordinated to poly(vinylpyrrolidone): isotropic to anisotropic transformation and morphology," Journal of Materials Science, vol. 46, no. 21, pp. 6988-6997, 2011.

[36] A. A. Bogdanov Jr., S. Gupta, N. Koshkina et al., "Gold nanoparticles stabilized with MPEG-grafted poly(L-lysine): in vitro and in vivo evaluation of a potential theranostic agent," Bioconjugate Chemistry, vol. 26, no. 1, pp. 39-50, 2015.

[37] V. S. Murthy, J. N. Cha, G. D. Stucky, and M. S. Wong, "Chargedriven flocculation of poly(l-lysine) - gold nanoparticle assemblies leading to hollow microspheres," Journal of the American Chemical Society, vol. 126, no. 16, pp. 5292-5299, 2004.

[38] V. Z. Prokopović, C. Duschl, and D. V. Volodkin, "Hyaluronic acid/poly-L-lysine multilayers coated with gold nanoparticles: cellular response and permeability study," Polymers for Advanced Technologies, vol. 25, no. 11, pp. 1342-1348, 2012.

[39] W. Sun, Q. Dai, J. G. Worden, and Q. Huo, "Optical limiting of a covalently bonded gold nanoparticle/polylysine hybrid material," The Journal of Physical Chemistry B, vol. 109, no. 44, pp. 20854-20857, 2005.

[40] K. Marycz, M. Marędziak, A. Śmieszek, J. Grzesiak, and A. Siudzińska, "The autologous gelsolin combined with exogenous nucleotides enhance chondrogenic differentiation in equine adipose derived mesenchymal stromal cells-an in vitro research," Journal of Cell Science \& Therapy, vol. 5, no. 4, 2014. 
[41] A. Frizziero, E. Giannotti, F. Oliva, S. Masiero, and N. Maffulli, "Autologous conditioned serum for the treatment of osteoarthritis and other possible applications in musculoskeletal disorders," British Medical Bulletin, vol. 105, no. 1, pp. 169184, 2013.

[42] G. F. Guidetti, A. Consonni, L. Cipolla, P. Mustarelli, C. Balduini, and M. Torti, "Nanoparticles induce platelet activation in vitro through stimulation of canonical signalling pathways," Nanomedicine: Nanotechnology, Biology, and Medicine, vol. 8, no. 8, pp. 1329-1336, 2012.

[43] J. Nicpoń, K. Marycz, J. Grzesiak, A. Śmieszek, and N. Y. Toker, "The advantages of autologus Adipose derived Mesenchymal Stem Cells (AdMSCs) over the Non-steroidal Antiinflammatory Drugs NSAIDs application for degenerative elbow joint disease treatment in dogs-twelve cases," Kafkas Üniversitesi Veteriner Fakültesi Dergisi, vol. 20, no. 3, pp. 345350, 2014.

[44] A. W. A. Baltzer, C. Moser, S. A. Jansen, and R. Krauspe, "Autologous conditioned serum (Orthokine) is an effective treatment for knee osteoarthritis," Osteoarthritis and Cartilage, vol. 17, no. 2, pp. 152-160, 2009.

[45] D. D. Frisbie, C. E. Kawcak, N. M. Werpy, R. D. Park, and C. W. Mcllwraith, "Clinical, biochemical, and histologic effects of intra-articular administration of autologous conditioned serum in horses with experimentally induced osteoarthritis," The American Journal of Veterinary Research, vol. 68, no. 3, pp. 290-296, 2007.

[46] M. B. Goldring, "Osteoarthritis and cartilage: the role of cytokines," Current Rheumatology Reports, vol. 2, no. 6, pp. 459465, 2000.

[47] N. T. K. Thanh, N. Maclean, and S. Mahiddine, "Mechanisms of nucleation and growth of nanoparticles in solution," Chemical Reviews, vol. 114, no. 15, pp. 7610-7630, 2014.

[48] A. Roffi, G. Filardo, E. Assirelli et al., "Does platelet-rich plasma freeze-thawing influence growth factor release and their effects on chondrocytes and synoviocytes?" BioMed Research International, vol. 2014, Article ID 692913, 10 pages, 2014. 

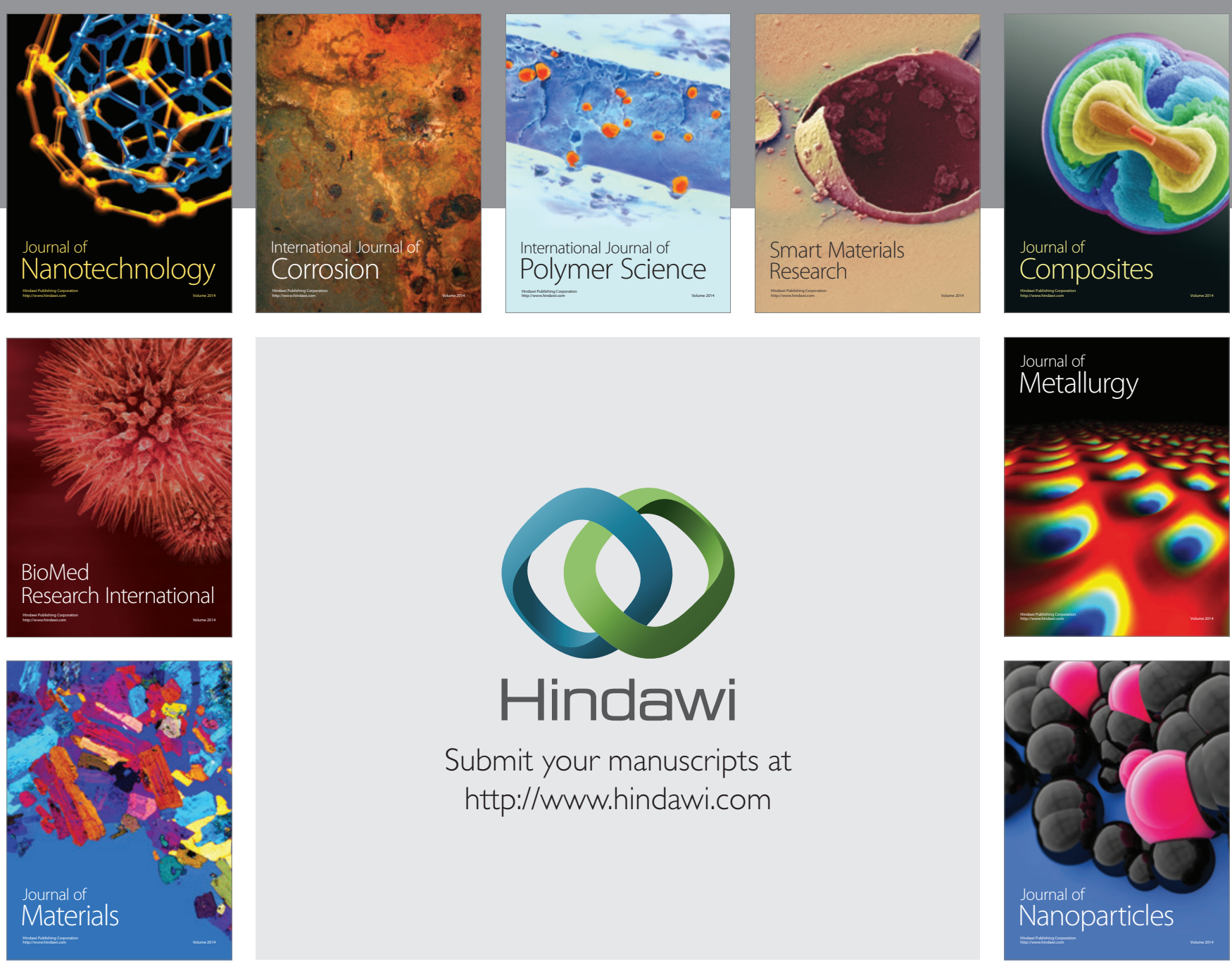

Submit your manuscripts at http://www.hindawi.com
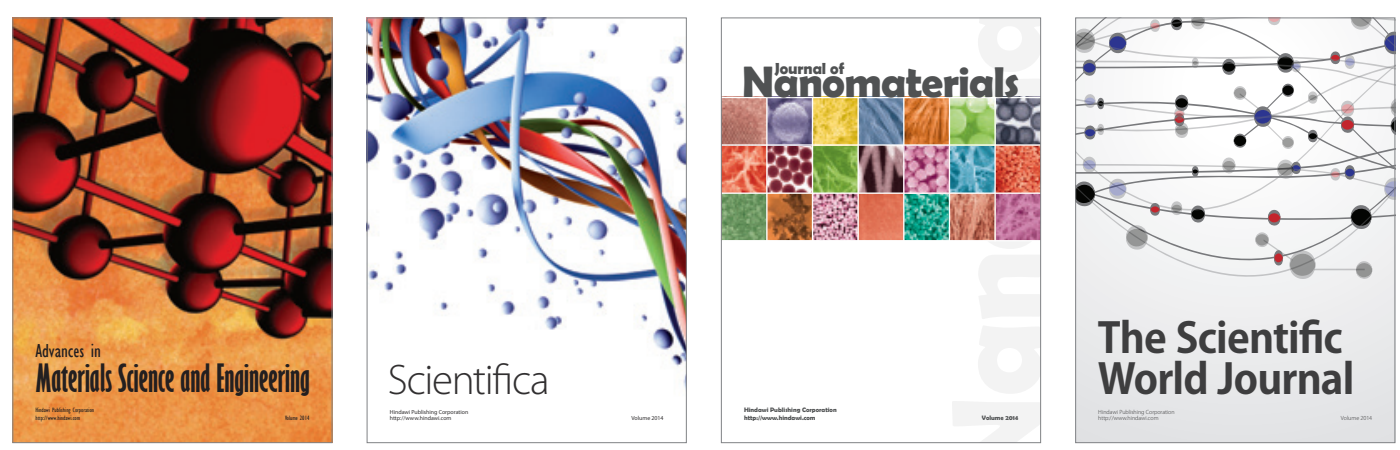

\section{The Scientific World Journal}
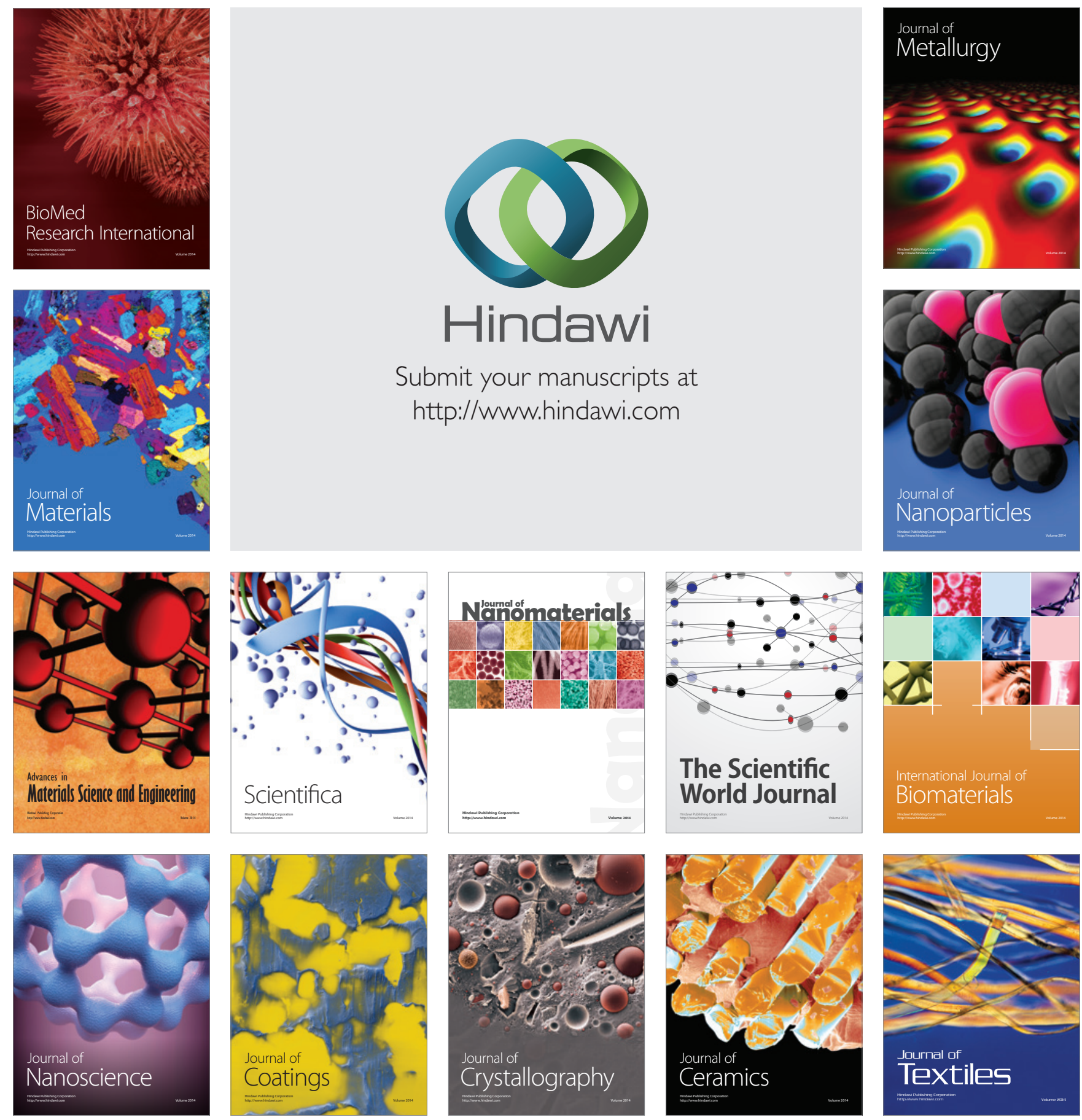